\title{
Prognostic value of interim PET/CT scan results in pediatric Hodgkin Lymphoma
}

\author{
Mohyeldin Abdelhalim ${ }^{1}$, Nadia Eldeeb ${ }^{1}$, Omar Zahra ${ }^{1}$, Amr Abdelkerim ${ }^{1}$, and Shady \\ Fadel $^{1}$
}

${ }^{1}$ Alexandria University

September 28, 2020

\begin{abstract}
Background: Treating Hodgkin lymphoma (HL) among children comprises a trade-off between cure and reduction of radiotherapy (RT) toxicity. Fluorodeoxyglucose positron emission tomography combined with computed tomography (PET/CT) identifies patients showing early favorable response to chemotherapy, and therefore can preclude the RT need. Objective: The current study aims to discuss the prognostic value of interim PET/CT following two cycles of chemotherapy OEPA (Oncovin, Etoposide, Prednisone, Adriamycin) in pediatric HL in terms of event-free survival (EFS) and overall survival (OS). Materials and Methods: We retrospectively included all HL patients younger than 18 years, between January 2010 and December 2017. Interim PET/CT was performed after two chemotherapy cycles. Patients were stratified into three risk groups into: group 1, group 2 and group 3. OEPA regimen was used in early disease while Cyclophosphamide, Oncovin, Prednisone and Dacarbazine were added in advanced disease. RT was avoided in all patients who achieved complete response (CR) by interim PET/CT. Results: 65 patients were included. Based on the negative interim PET/CT responses, 43 (66.1\%) patients were treated without RT. The 5-year EFS for the entire cohort was $89 \%$ and OS was $97 \%$. Most of the PET/CT scans at the end of treatment were done in positive interim PET/CT cases, while in early responders; only $\mathrm{CT}$ scans were done. Conclusions: Interim PET/CT in pediatric HL is a good prognostic tool to evaluate early responders after the 2 initial chemotherapy cycles and hence omit RT to this group of patients.
\end{abstract}

\section{Introduction}

Hodgkin lymphoma (HL) constitutes around $6 \%$ of yearly overall childhood cancer in USA and ranks as the most common malignancy among adolescents between 15 and 19 years ${ }^{(1,2)}$. Since the late 1960s and thereafter, attention was undertaken to achieve a balance between attaining adequate response to combined modalities (including intensified doses of chemotherapy while eliminating the gonadotoxic regimens) and reduction of radiotherapy (RT) dose and field, in order to reduce the long-term adverse events among the survivors. Therefore, considerable efforts were conducted to identify predictive and prognostic factors to reduce management burden without producing significant survival failures ${ }^{(3)}$. Definitions for interim response are usually protocol specific. It may vary from (2-dimensional) size reductions of more than $50 \%$, amounting to a complete anatomical or metabolic response by conventional imaging or Fluorodeoxyglucose positron emission tomography combined with computed tomography $(\mathrm{PET} / \mathrm{CT})^{(4)}$. Throughout the literature, many published studies have assessed the role of interim response to titrate further therapeutic modalities. The Pediatric Oncology Group adopted a response-based therapy approach for intermediate and advanced stages by applying dose-dense ABVE-PC (doxorubicin, bleomycin, vincristine, etoposide, prednisone, and cyclophosphamide) combined with 21 Gy involved-field radiation therapy (IFRT) ${ }^{(5)}$. The dose-dense regimen allowed reduction in exposure to chemotherapy in $63 \%$ of children who attained a rapid early anatomical response on $\mathrm{CT}$ after three cycles of ABVE-PC ${ }^{(5)}$. With the same RT dose (21 Gy), the five-year event-free survival (EFS) was comparable for rapid early responders (treated with three cycles of ABVE-PC) and for the slow 
early responders (who received five cycles of the same regimen) [86\% versus $83 \%$ respectively] ${ }^{(5)}$.

In a newly published report, by the Children Oncology Group (AHOD0831), patients with higher stages (IIIB / IVB) received two cycles of ABVE-PC. Rapid early responders were consolidated with another 2 cycles of the same regimen whereas slow early responders (SER) received two cycles of ifosfamide/vinorelbine and ABVE-PC. Only Patients with initially bulky tumors and/or those with SER received further RT . The fouryear EFS of the entire cohort was $91.9 \%{ }^{(6)}$. Most European centers are adopting the protocols brought in by European Network for Pediatric Hodgkin Lymphoma (EuroNet- PHL), which came out in $2007^{(6)}$. Based on the EuroNet- PHL, all first line patients received two chemotherapy cycles of OEPA (Oncovin, Etoposide, Prednisone, Adriamycin) and then pursued a response assessment by PET/CT (preferably until day 17 ) after the last chemotherapy treatment, i.e. d28 -31 of the second cycle of OEPA ${ }^{(7)}$.

Patients are stratified into three treatment groups: 1) TG-1: patients of stages I A/B and II A without bulk $>=200 \mathrm{ml}$ and without ESR $>=30 \mathrm{~mm} / \mathrm{hr}$. 2) TG-2: patients of stages IEA/B, IIEA, II B or III A and patients of stages I A/B and II A with bulk $>=200 \mathrm{ml}$ and/or ESR $>=30 \mathrm{~mm} / \mathrm{hr}$. 3) TG-3: patients of stages IIEB, IIIEA/B, III B or IV A/B. ${ }^{(7)}$ Patients in TG-1 do not receive more chemotherapy, while TG-2 and TG-3 patients receive additional two or four COPDAC (Cyclophosphamide, Vincristine, Prednisone, Dacarbazine) cycles respectively based on the GPOH- HD 2002 Pilot study ${ }^{(7)}$. We herein tried to study the role interim PET/CT after two cycles of chemotherapy to preclude the need for further RT.

\section{Methods}

The study had been approved by the research and ethical committees of the contributing hospitals. We retrospectively enrolled all patients aged less than 18 years, treated for HL in our university hospital and two other national cancer institutes in our province between January 2010 and December 2017 and to whom PET/CT was obtained after two cycles of chemotherapy. Chemotherapy protocol involved Vincristine, Etoposide, Prednisone and Adriamycin. Those who have achieved complete response (CR) after chemotherapy didn't receive RT. We ended with a total of 65 patients, whose records have been analyzed for demographic data, history details, clinical examination findings notably nodal and extra nodal involvement, laboratory investigations, diagnostic tool to confirm the diagnosis of HL, management regimen, and follow-up.

Statistical Analysis

Data were fed to the computer and analyzed using IBM SPSS software package version 20.0. (Armonk, NY: IBM Corp). Qualitative data were described using number and percent. Quantitative data were described using range (minimum and maximum), mean, and standard deviation. Significance of the obtained results was judged at the $5 \%$ level. The used tests were: Chi-square test, Fisher's Exact, Student t-test, Mann Whitney test and Regression.

\section{Results}

The present study included 65 pediatric patients with HL treated in our institutes with the Euronet protocol. All patients were not initially staged with PET/CT. Interim PET/CT scan was obtained for all patients after 2 OEPA cycles. At the end of treatment, PET/CT was done for 37 patients (55.3\%), whereas 29 patients $(44.6 \%)$ with initial negative interim PET/CT were examined with only regular CT scan. CR on interim PET/CT was achieved in 66.1\% [43 out of 65 patients] and therefore RT was omitted in this group of patients.

Median Follow-up, 5-year EFS and overall survival (OS) of the entire cohort

The median follow up period for the 65 pediatric HL patients in the study was 59 months. The 5 -year EFS for the entire cohort was $89 \%$ (Figure (1)), and OS was $97 \%$ (Figure (2)).

Assessment of the event-free survival

Events were more frequently encountered among patients who received RT as they didn't achieve CR (22.7\% [5/22 patients] versus $4.7 \%[2 / 43]$ of patients with $\mathrm{CR}$ and who skipped the RT). This was statistically 
significant $(\mathrm{P}=0.035)$, Figure (3), Figure (4). Encountered events at both groups were as follow: Five events among 22 patients who underwent RT ( recurrences $(n=2)$ and RT -related rise in thyroid stimulating hormone $(\mathrm{n}=3)$ ) and solely two events in second group who skipped the RT(recurrence in one patient who died after transplantation, whereas the second patient died during chemotherapy cycles due to Transfusion Reaction Acute Lung Injury (TRALI).

Assessment of the overall survival

We had only one patient who died among the 22 who achieved CR (and hence didn't receive RT) and one patient who died among the 45 who didn't achieve CR (2.3\% vs. $4.5 \%)$. These results were not statistically significant in terms of $\mathrm{OS},(\mathrm{P}=1)$, Figure (5), Figure (6).

\section{Discussion}

A combined modality approach in pediatric HL usually results in excellent response rates and OS ${ }^{(8)}$. Though, the survivors might experience RT related adverse effects (such as secondary malignancies, cardiovascular dysfunction, and hormonal disorders $)^{(9)}$. Achieving a cure without long term toxicity compels the recognition of those patients who can be treated with no RT while conserving their OS. A vigorous management approach based on risk stratification as well as on an early favorable response to chemotherapy is required (10). In this retrospective study, 65 pediatric patients with HL were managed according to the protocol of European Network for Pediatric Hodgkin Lymphoma (EuroNet- PHL). All enrolled patients were not assessed by $\mathrm{PET} / \mathrm{CT}$ prior to treatment. The Lugano classification endorses the value of PET/CT for initial staging of HL being more sensitive than CT alone, shifting the stage of about 10 to $30 \%$ of patients towards an upgrading rather than a downgrading. Though, the use of initial PET/CT did not have an implication on the management plane or outcome ${ }^{(11)}$. We assessed the initial response after 2 OEPA chemotherapy cycles with interim 18F- fluorodeoxyglucose PET/CT (18F-FDG) and provided additional RT for those who failed to achieve CR. Further COPDAC chemotherapy cycles were delivered conforming to their initial treatment group classification. Most of the children were included in TG-2 (41.5\%), while those in TG-1 and TG-3 were $24.6 \%$ and $33.9 \%$ respectively. The median follow-up period in our series was 59 months, and we achieved a 5-year OS and 5-year EFS of $97 \%$ and $89 \%$ respectively. This largely concur with the results of Children's Oncology Group study AHOD0031 encompassing 1712 child with HL treated with two chemotherapy cycles of ABVE-PC regimen (doxorubicin, bleomycin, vincristine, etoposide, prednisone, and cyclophosphamide) followed by interim assessment by PET/CT. They showed four year survival and free event of $97.8 \%$ and $87.9 \%^{(12)}$. PET/CT obtained early during treatment of HL in adults showed a high prognostic value ${ }^{(13)}$. In a retrospective multicenter study of 260 patients who had an interim PET/CT after two cycles of ABVD, the 3 year progression free survival of patients with positive and negative scans were $28 \%$ and $95 \%^{(14)}$. In our study, events were more frequently encountered among those who failed to achieve CR and received RT (22.7 $\%$ versus $4.7 \%$ of those with $\mathrm{CR}$ without $\mathrm{RT}$ ). This was statistically significant in terms of EFS ( $\mathrm{P}=0.035)$. Similar outcomes were reported as in Metzger et al ${ }^{(15)}$ who conducted a Multi-institutional, nonrandomized trial including 88 patients with favorable-risk HL to identify the need for RT after an early response to chemotherapy. 47 patients achieved CR and RT was not given, while 41 received 25.5 Gy-involved-field RT after an incomplete response to VAMP (vincristine, amethopterine, methotrexate, and prednisone). The 2-year EFS among both groups were $89.4 \%$ and $92.5 \%$ and was not statistically significant $(\mathrm{P}=0.61)^{(15)}$.

The Society for Pediatric Oncology and Haematology (GPOH-HD-2002) had a nearly comparable outcome to ours. They enrolled 573 patients, with median observation time over 58.6 months. The 5 -year OS and EFS were $97.4 \%$ and $89.0 \%$, respectively ${ }^{(16)}$. In TG-1, overall EFS was $92.0 \%$. EF of patients without RT $(93.2 \%)$ was similar to that of irradiated patients (91.7\%). In TG-2 and 3, EFS did not markedly vary between boys and girls $\left(90.2 \%\right.$ v $84.7 \%$, respectively; P .12) ${ }^{(17)}$. Their CR criteria were strict to the reduction of more or equal to $95 \%$ and equal or less than $2 \mathrm{~mL}$ of the initial volume ${ }^{(16)}$. We have successfully managed $66.1 \%$ of our cases omitting RT, across all treatment groups, on the basis of interim PET response. An even better result was published by Mauz-Korholz et al ${ }^{(16)}$ who achieved favorable results in $80 \%$ of their 49 patients. They referred to a protocol of doxorubicin, bleomycin, vinblastine, dacarbazinebased regimen in early disease and cyclophosphamide, vincristine, prednisolone, procarbazine, doxorubicin, 
bleomycin, vinblastine-based regimen in advanced disease $\mathrm{e}^{(16)}$.

On the other side, The Children's Cancer Group trial CCG 5942 published a long-term study with median follow up period of 7.7 years, enrolling 498 patients who reached initial CR after receiving COPP/ABV hybrid chemotherapy ${ }^{(17)}$. They were randomly distributed to obtain either RT or no subsequent treatment (17). The 10-year EFS was significantly better for those who received RT (91.2\% vs. $82.9 \%$ ), yet the OS did not vary remarkably $(97.1 \% \text { vs. } 95.9 \%)^{(17)}$. The choice of chemotherapy may also dictate the need for RT. It has been shown that a more aggressive chemotherapy regimen may offset the potential benefit of the addition of RT. This may explain why it was more difficult to show a statistical improvement in EFS with IFRT in clinical group 2 and 3 patients in this study who received more intensive chemotherapy. Also, small numbers may not yield adequate power to detect a true difference; especially for group 3.The issue is how to determine which approach will produce the least toxicity, as both chemotherapy and low-dose RT are associated with risks ${ }^{(18)}$. We had mortality among those who skipped the RT (4.6\%) and also among those who achieved and those who didn't get CR $(2.3 \%$ and $4.5 \%$ respectively. There was no statistical significance in terms of OS across all the parameters and the variables due to the high cure rate following therapies.

\section{Limitations}

Despite enrolling a relatively good number of HL patients in the study, its retrospective nature remains a major limitation. One such limitation is the inability to initially stage all the patients with PET/CT.

\section{Conclusions}

Interim PET/CT scan in pediatric HL is a good prognostic tool to evaluate early responders after the 2 initial chemotherapy cycles and hence omit RT to this group of patients. Late RT adverse effects as secondary malignancies, cardiac and endocrine abnormalities among survivors in patients achieving $\mathrm{CR}$ after interim PET/CT could be avoided. The 5-year EFS rate estimates in patients with CR after 2 OEPA treated without RT is about $89 \%$.

\section{Figure Legends}

Figure 1. EFS of the entire cohort.

Figure 2. OS of the entire cohort.

Figure 3. Effect of interim PET-CT results on EFS.

Figure 4. Effect of RT received on EFS.

Figure 5. Effect of interim PET-CT results on OS.

Figure 6. Effect of RT received on OS.

\section{References}

1. Allen CE, Kelly KM, Bollard CM. Pediatric lymphomas and histiocytic disorders of childhood. Pediatr Clin North Am 2015; 62(1):139-65.

2. Sherief LM, Elsafy UR, Abdelkhalek ER, Kamal NM, Elbehedy R, Hassan TH, et al. Hodgkin lymphoma in childhood: clinicopathological features and therapy outcome at 2 centers from a developing country. Medicine (Baltimore) 2015; 94(15):e670.

3. Bonadonna G, Santoro A. ABVD chemotherapy in the treatment of Hodgkin's disease. Cancer Treat Rev 1982; 9(1):21-35.

4. Kelly KM, Sposto R, Hutchinson R, Massey V, McCarten K, Perkins S, et al. BEACOPP chemotherapy is a highly effective regimen in children and adolescents with high-risk Hodgkin lymphoma: a report from the Children's Oncology Group. Blood 2011; 117(9):2596-603. 
5. Schwartz CL, Constine LS, Villaluna D, London WB, Hutchison RE, Sposto R, et al. A risk adapted, response-based approach using ABVE- PC for children and adolescents with intermediate- and high-risk Hodgkin lymphoma: the results of P9425. Blood 2009; 114(10):2051-9.

6. Kelly KM, Cole PD, Pei Q, Bush R, Roberts KB, Hodgson DC, et al. Response-adapted therapy for the treatment of children with newly diagnosed high risk Hodgkin lymphoma (AHOD0831): a report from the Children's Oncology Group. Br J Haematol 2019; 187(1):39-48.

7. Hudson MM, Krasin MJ, Kaste SC. PET imaging in pediatric Hodgkin's lymphoma. Pediatr Radiol $2004 ; 34(3): 190-8$.

8. Totadri S, Radhakrishnan V, Ganesan TS, Ganesan P, Kannan K, Lakshmipathy KM, et al. Can Radiotherapy Be Omitted in Children With Hodgkin Lymphoma Who Achieve Metabolic Remission on Interim Positron Emission Tomography? Experience of a Tertiary Care Cancer Referral Center. J Glob Oncol 2018; 4:1-7.

9. Radford J, Longo DL. Second Cancers after Treatment for Hodgkin's Lymphoma-Continuing Cause for Concern. N Engl J Med2015; 373(26):2572-3.

10. Connors JM. Risk assessment in the management of newly diagnosed classical Hodgkin lymphoma. Blood 2015; 125(11):1693-702.

11. Cheson BD, Fisher RI, Barrington SF, Cavalli F, Schwartz LH, Zucca E, et al. Recommendations for initial evaluation, staging, and response assessment of Hodgkin and non-Hodgkin lymphoma: the Lugano classification. J Clin Oncol 2014; 32(27):3059-68.

12. Stein H, Marafioti T, Foss HD, Laumen H, Hummel M, Anagnostopoulos I, et al. Down regulation of BOB.1/OBF.1 and Oct2 in classical Hodgkin disease but not in lymphocyte predominant Hodgkin disease correlates with immunoglobulin transcription. Blood 2001; 97(2):496-501.

13. Hutchings M, Loft A, Hansen M, Pedersen LM, Buhl T, Jurlander J, et al. FDG-PET after two cycles of chemotherapy predicts treatment failure and progression-free survival in Hodgkin lymphoma. Blood 2006; 107(1):52-9.

14. Gallamini A, Barrington SF, Biggi A, Chauvie S, Kostakoglu L, Gregianin M, et al. The Predictive Role of Interim Positron Emission Tomography for Hodgkin Lymphoma Treatment Outcome Is Confirmed Using the Interpretation Criteria of the Deauville Five-Point Scale. Haematologica 2014; 99(6):1107-13.

15. Metzger ML, Weinstein HJ, Hudson MM, Billett AL, Larsen EC, Friedmann A, et al. Association between radiotherapy vs no radiotherapy based on early response to VAMP chemotherapy and survival among children with favorable-risk Hodgkin lymphoma. JAMA 2012;307:2609-16.

16. Mauz-Korholz C, Hasenclever D, Dorffel W, Ruschke K, Pelz T, Voigt A, et al. Procarbazine-free OEPA-COPDAC chemotherapy in boys and standard OPPA-COPP in girls have comparable effectiveness in pediatric Hodgkin's lymphoma: the GPOH-HD-2002 study. J Clin Oncol 2010; 28(23):3680-6.

17. Wolden SL, Chen L, Kelly KM, Herzog P, Gilchrist GS, Thomson J, et al. Long-term results of CCG 5942: a randomized comparison of chemotherapy with and without radiotherapy for children with Hodgkin's lymphoma-a report from the Children's Oncology Group. J Clin Oncol 2012; 30(26):3174-80. 

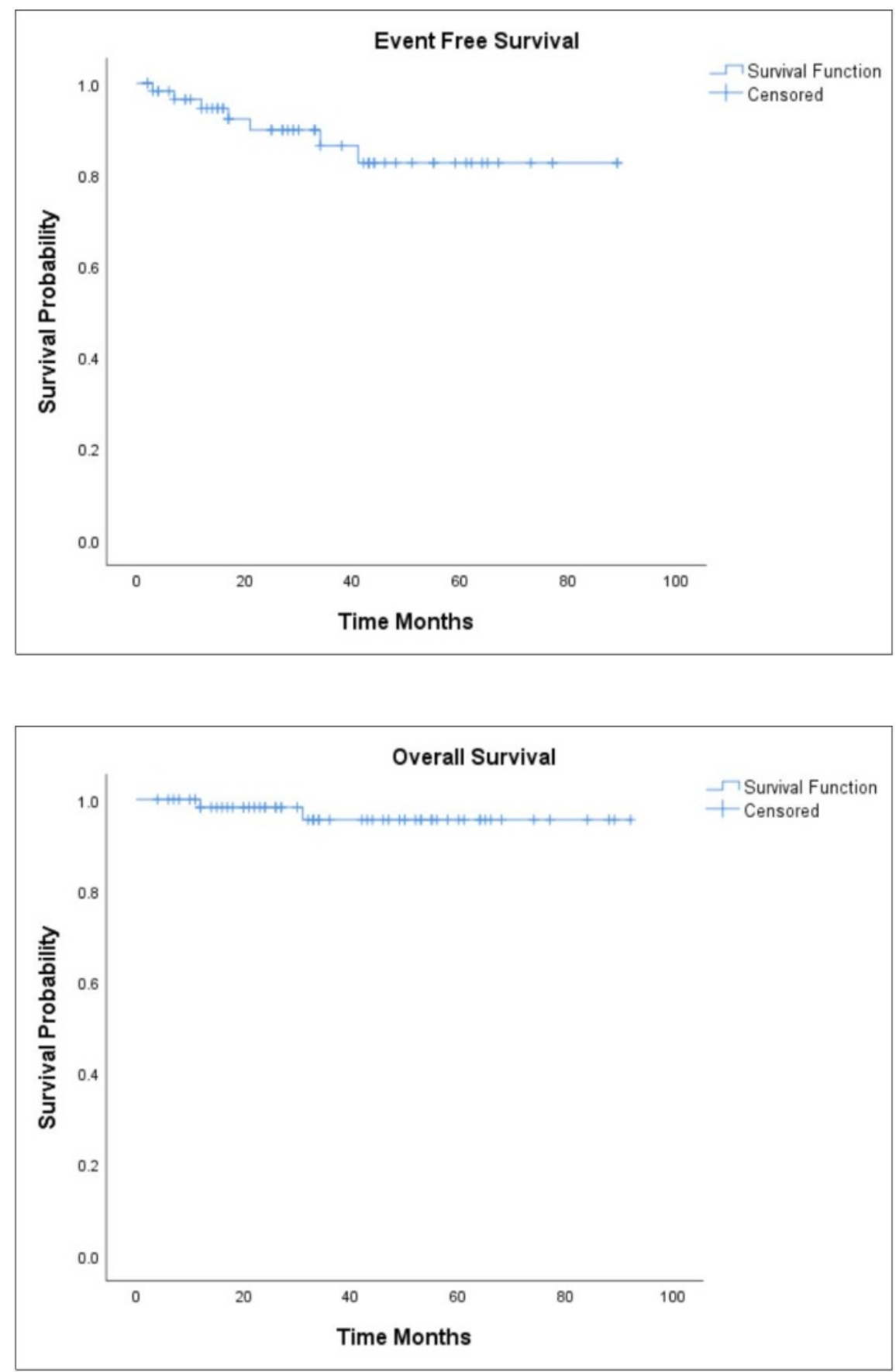

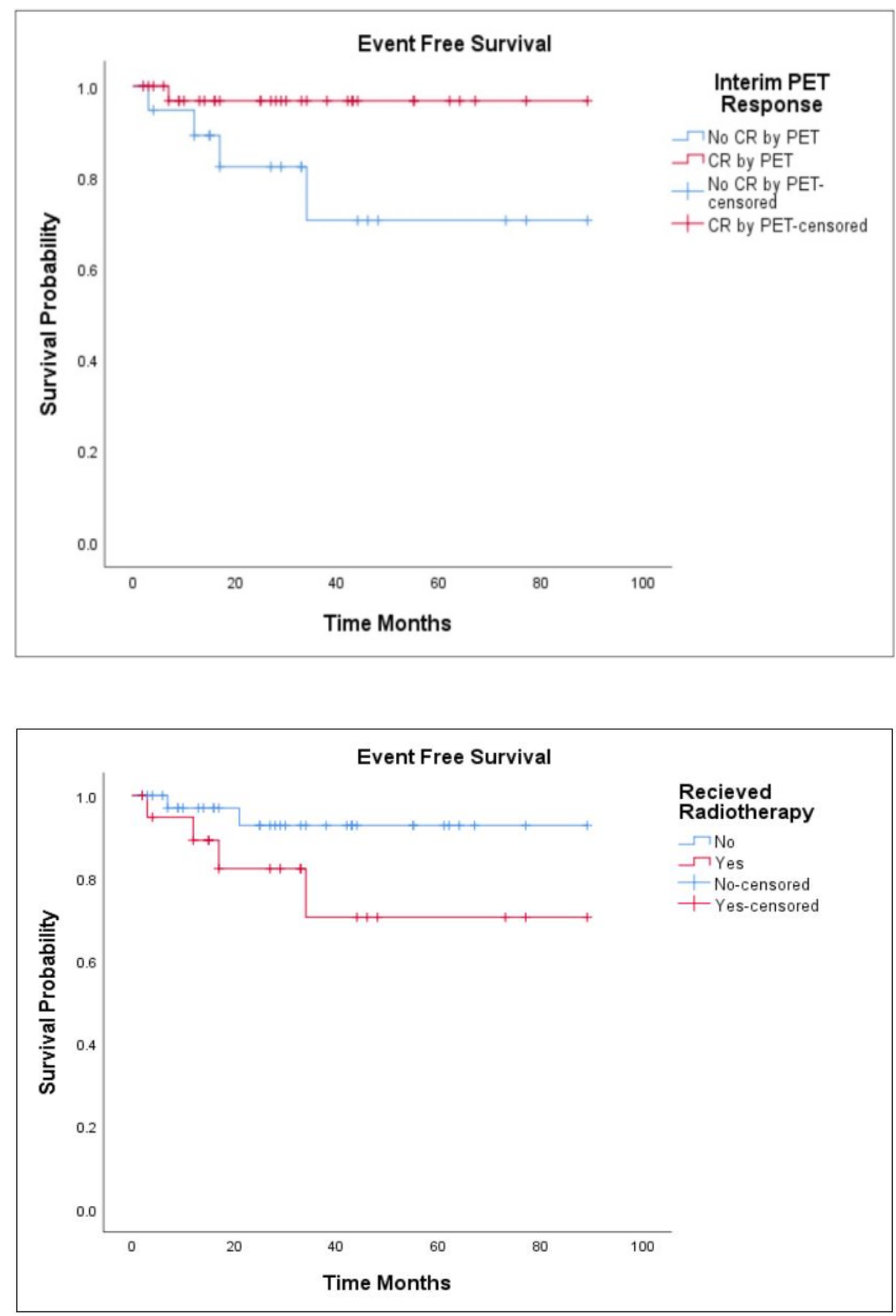

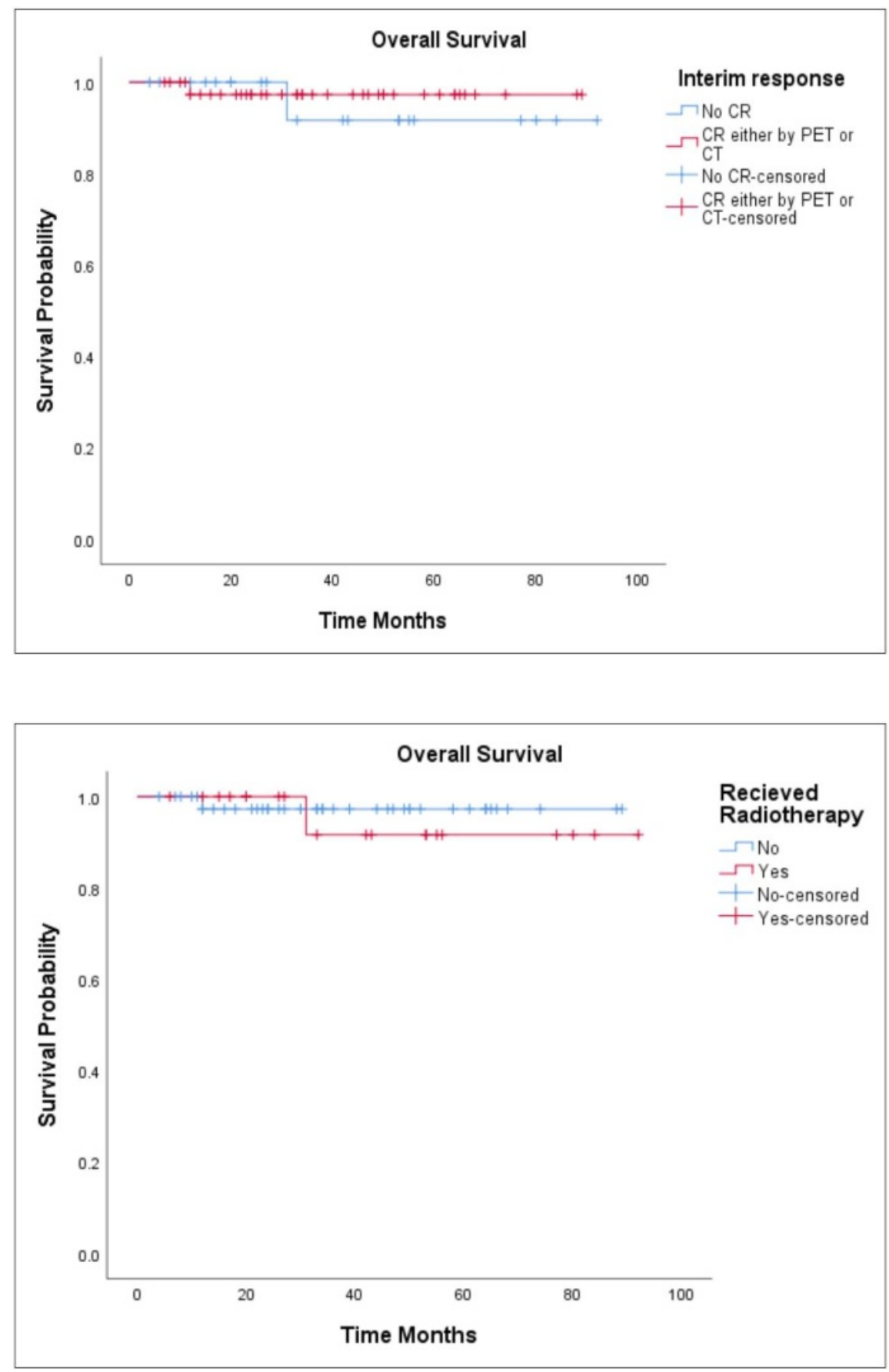